\title{
Laser scanning microscopy: seeing more by imaging less.
}

\author{
N.S. White
}

Oxford University, Sir William Dunn School of Pathology (Microscopy Lab), Oxford. UK. OX1 3RE nick.white@path.ox.ac.uk

Conventional fluorescence microscopy results in all points of a sample being imaged simultaneously to a complete representation in a wide field of view. Illumination is not focused but bathes the whole specimen. The laser scanning microscope (LSM), has advantages by not adhering to this model.

In a non-confocal single-photon LSM, continuous laser illumination is focused (imaged) into a specimen. In-focus and out-of-focus fluorescence are not imaged but integrated by a large detector into a single measurement. The optical probe is scanned through the sample to buildup a 2-D picture. The imaging components determine the instrument resolution. Thus the shorter wavelength of the simple LSM illumination results in superior resolution to the longer fluorescence emission image of the conventional system. This has important consequences for multi-photon configurations.

In the Confocal (C)LSM, fluorescence from the focused laser at each sequential point in the scan is imaged via a small aperture by a 'point' detector. The aperture blocks outof-focus light, resulting in optical sectioning. The detection is no longer wide-field, and the imaged spot means that the fluorescence wavelength must now be considered in addition to the excitation when determining resolution. Fortunately, the combination of two imaging components(in this case excitation and detection optics) gives an additional 1.4x increase in resolution (approx) and so the CLSM can deliver superior resolution to the conventional and simple LSM cases. This result also has implications for multi-photon configurations.

The MPLSM uses an optical probe confined to a small volume at the focus of a lens. This 3D probe is derived entirely from near-infrared laser illumination, in contrast to confocal (C-) LSM that combines illumination and detection optics for a similar result.

By increasing the laser power and operating at two (or three) times the usual excitation wavelength, multiple photons may be absorbed simultaneously by each fluorescent molecule. Excitation efficiency then increases by the $2^{\text {nd }}$ or $3^{\text {rd }}$ power, respectively, of the laser intensity and so only happens near the focus. An ultra-fast pulsed laser $(\sim 100 \mathrm{fs} @ 100 \mathrm{MHz})$ is needed for a high peak intensity but low time-averaged power to prevent specimen damage. With this multiphoton excitation no out-of-focus fluorescence is generated, so no confocal aperture is needed for optical sectioning. The simple LSM configuration described above can then provide maximum detection efficiency. The 2-photon process provides the equivalent of the dualfocussed excitation and detection of confocal microscopy in delivering a $\sqrt{ } 2$ increase in resolution compared to a conventional microscope operating at the same wavelength. 3-photon excitation can show a cuberoot improvement. Unfortunately the resolution, as in the simple non-confocal case, is dependent on 
the excitation laser wavelength - i.e. in the near IR for 2- and 3-photon excitation, and is intrinsically worse than both the previous LSM examples under ideal conditions. The major benefits of MPLSM can, however, be realized when collecting data under non-ideal conditions.

Although the ultra-fast laser pulses used for multi-photon excitation have a wider bandwidth than continuous single-photon excitation, chromatic aberrations are substantially reduced with largearea detectors. Monochromatic corrections (such as spherical) can be incorporated more easily (even automated) in the excitation optics.

By exciting the fluorescence only in a localized volume around the focus, photodamage away from the optical section is avoided. This is in contrast to the single-photon cases where unwanted out-offocus planes are always excited (and thus subject to photo-damage) but the resultant fluorescence just degrades the image or is discarded in the confocal case by the detectoraperture.

For thick specimens that scatter light significantly we can further exploit the multiphoton configuration. As scattering losses decrease at longer wavelengths, nearIR illumination can penetrate significantly further than the shorter wavelengths used for single-photon excitation. However effective the IR light is at penetrating the specimen it still remains to collect the emerging fluorescence that will also be scattered by the sample. By removing the requirement to image the fluorescing spot onto a confocal aperture, the detection optics can be made to collect scattered light more effectively when focused deep within the specimen.

In order to assess the relative merits of these (and other) LSM configurations for different specimens, it is desirable to separate the many image-degrading processes into simple reproducible measurements. These can readily be achieved using some simple reflection and fluorescence test samples. SPR (surface plasmon resonance) signal from small gold particles offers an additional well-defined non-bleachable test specimen for some critical measurements. 